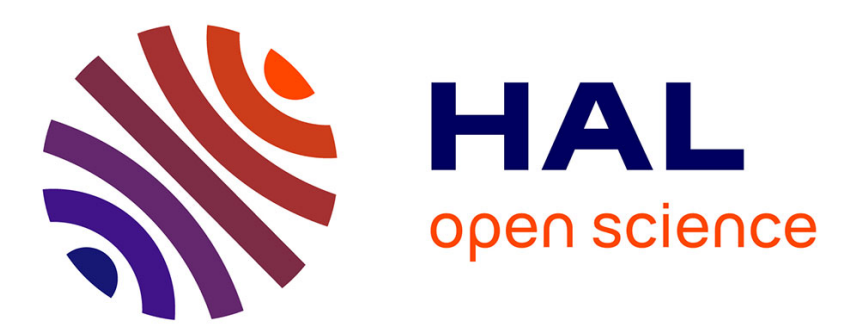

\title{
LOS RASGOS DE PERSONALIDAD MEDIDOS CON EL MMPI-II COMO PREDICTORES DEL RENDIMIENTO ACADÉMICO EN ESTUDIANTES UNIVERSITARIOS
}

Rodolfo Sergio González Castro

\section{To cite this version:}

Rodolfo Sergio González Castro. LOS RASGOS DE PERSONALIDAD MEDIDOS CON EL MMPIII COMO PREDICTORES DEL RENDIMIENTO ACADÉMICO EN ESTUDIANTES UNIVERSITARIOS. ALTAmira Revista Academica, 2015, 10, pp.24-37. 10.15418/altamira10-002 . hal01179582

\author{
HAL Id: hal-01179582 \\ https://hal.science/hal-01179582
}

Submitted on 1 Aug 2015

HAL is a multi-disciplinary open access archive for the deposit and dissemination of scientific research documents, whether they are published or not. The documents may come from teaching and research institutions in France or abroad, or from public or private research centers.
L'archive ouverte pluridisciplinaire HAL, est destinée au dépôt et à la diffusion de documents scientifiques de niveau recherche, publiés ou non, émanant des établissements d'enseignement et de recherche français ou étrangers, des laboratoires publics ou privés.

\section{(ㄷ)(1) $\$$}

Distributed under a Creative Commons Attribution - NonCommerciall 4.0 International 


\title{
LOS RASGOS DE PERSONALIDAD MEDIDOS CON EL MMPI-II COMO PREDICTORES DEL RENDIMIENTO ACADÉMICO EN ESTUDIANTES UNIVERSITARIOS
}

\author{
Rodolfo Sergio González Castro * \\ * Psicólogo y Doctor en Educación. Maestro de Tiempo Completo e Investigador de la Universidad de \\ Tijuana. Tijuana México. Email: rodolfogonzalezcastro@gmail.com Teléfono 526646879411. \\ Publicado en ALTAmira Revista Académica de la Universidad de Tijuana. Número 10. Julio- \\ Septiembre 2015. Páginas 24-37. Fecha de Envío: 12 de febrero del 2015. Fecha de aprobación: \\ 1 de julio 2015. ISSN: 2007-8854 DOI: 10.15418/altamira10-002
}

\section{RESUMEN}

Investigación aplicada en la que se sometió a prueba la hipótesis de que los rasgos de personalidad medidos mediante el Inventario Multifacético de Personalidad de Minnesota (MMPI-II) pueden ser utilizados para predecir el rendimiento académico en alumnos de licenciatura. Los resultados obtenidos indican que dicho inventario resulta ineficiente en conjunto (4.65\% de eficacia) para diagnosticar e identificar que alumnos tienen o tendrán problemas de desempeño académico durante su estancia en la universidad, además se corroboró que los factores contextuales como la actividad mental de los estudiantes tienen mayor peso explicativo en el rendimiento escolar que los rasgos de personalidad.

Tags: rasgos, personalidad, mmpi-2, predicción, rendimiento, investigación, estudiantes, universitarios, diagnóstico, eficacia.

\section{INTRODUCCIÓN}

Las instituciones de educación superior privadas se enfrentan hoy en día ante la necesidad de fortalecer su matrícula mediante normas y estándares más flexibles en sus procesos de selección, lo que propicia la aceptación de una cantidad considerable de alumnos con un historial previo de bajo y mediano rendimiento (secundaria y bachillerato), pero en contra parte, obtienen un registro muy bajo de universitarios con alto desempeño escolar.

Adicionalmente, en ocasiones dichas universidades no cuentan con las herramientas de diagnóstico adecuadas que les permitan la implementación de programas de nivelación académica para sus estudiantes de bajo perfil, lo que tiene como consecuencia un incremento en sus estadísticas de reprobación, bajo promedio general de aprovechamiento de sus alumnos, egresados de bajo perfil profesional y en los casos más graves se incrementan considerablemente los alumnos que se dan de baja de sus estudios universitario por causas académicas. 
En el caso de los instrumentos de diagnóstico, una de las pruebas mas utilizadas por las universidades es el Inventario Multifacético de Personalidad de Minnesota (MMPI-2), el cual es un cuestionario de 567 preguntas que requiere al examinado al menos una o dos horas de su tiempo, un supervisor capacitado en la prueba y un tiempo considerable de trabajo del evaluador para la calificación, obtención de las gráficas de los perfiles de personalidad y la interpretación de los resultados (Butcher, Lim y Nezami, 1998).

EI MMPI-II se ha utilizado en universidades públicas y privadas para normalizar la prueba en poblaciones latinoamericanas pero también para obtener el perfil psicológico o de personalidad de los estudiantes, para conocer la correlación entre el perfil de personalidad y el rendimiento académico de los universitarios y con frecuencia se aplica como instrumento de selección de alumnos de nuevo ingreso.

De hecho la versión revisada del MMPI-2 traducida al español, en su adecuación a la población mexicana, se aplicó a una muestra de universitarios de la UNAM, con el objeto de adaptar la prueba estableciendo normas y puntuaciones $\mathrm{T}$, además de obtener un análisis del funcionamiento de los reactivos con la población de nuestro país (Hathaway y McKinley, 1995).

Respecto a la obtención de perfiles psicológicos, tenemos por ejemplo el estudio efectuado por la Universidad de Guadalajara que aplicó el MMPI-II a sus alumnos de nuevo ingreso de las carreras de salud con el propósito de identificar los rasgos principales de su perfil de psicológico, para un posible uso educativo de estos datos en el futuro y, por otra parte para aportar elementos a la literatura sobre el tema acerca del funcionamiento y adecuación del inventario en poblaciones mexicanas normales (Martínez, Nava, Ortega, 2005).

En cuanto al rendimiento académico, en 1988 se efectuó una investigación sobre los rasgos de personalidad medidos por el MMPI-II en alumnos de nuevo ingreso a la Escuela de Ciencias Psicológicas de la Universidad San Carlos de Guatemala. En dicho estudió se encontraron rasgos de personalidad vinculados al bajo rendimiento y se recomendó "la creación de programas de apoyo con fines terapéuticos destinados a los estudiantes, para reforzar aquellos rasgos que puedan ayudarle a enfrentar adecuadamente su vida, y buscar los mecanismos más eficaces para el logro de una orientación del paciente en aquellos rasgos que por su naturaleza, le provoquen mayor grado de conflicto personal y social" (Lemus, 1988).

En 1991 se efectuó un estudio similar sobre los "Rasgos de personalidad del estudiante de primer ingreso de la Escuela de Ciencias Psicológicas según el MMPI y su relación con el rendimiento académico". El investigador concluyó que los rasgos de personalidad sí influían en el rendimiento académico de los estudiantes de primer ingreso, en particular encontró diferencias significativas en la escala $\mathrm{F}$ con respecto a bajo y alto rendimiento de los alumnos (De León, 1991).

Norma Coppari ha efectuado varias investigaciones con respecto a la utilización del MMPI-II en estudiantes universitarios en las que se ha buscado correlacionar su perfil de personalidad con su perfil vocacional (Coppari y Colbs, 2001), con sus patrones de comportamiento (Coppari y Colbs, 2007) y para efectuar un análisis multivariado de perfiles (Coppari, 2007), entre otros estudios. 
En México en el 2011 se realizó un estudio denominado "Factores asociados al rendimiento académico en alumnos de la Facultad de Medicina" en la Universidad Nacional Autónoma de México con el objetivo de identificar la influencia de las características de estudiantes en el programa de alta exigencia académica al final del primer año de la carrera en su rendimiento escolar. Dicho estudio encontró que el único predictor del rendimiento académico es el examen general de conocimientos y que el $19.7 \%$ de los estudiantes padecían algún tipo de psicopatología diagnosticada por el MMPI, principalmente depresión (Vargas y otros, 2011).

En el 2011, la Universidad de Tamaulipas aplicó el MMPI a los alumnos de nuevo ingreso de la licenciatura en Psicología, Enfermería, Seguridad, Seguridad y medio ambiente con propósitos descriptivos y como herramienta predictiva del comportamiento académico de dichos alumnos (Juana Medellín Moreno \& Salazar, 2011).

En un estudio efectuado en Guatemala por Zinzi Raquel García en el que se buscó correlacionar Rasgos de Personalidad y Éxito Académico en los alumnos de nuevo ingreso de la Universidad de San Carlos, solamente encontró que la escala Desviación Psicopática obtuvo un valor significativo para aceptar la hipótesis alternativa, en tanto el resto de escalas confirmaron la hipótesis nula (Ríos, 2012).

No siempre el MMPI ha arrojado resultado significativos. Por ejemplo en México se publicó en el 2003 un artículo titulado "Predicción del Rendimiento académico Universitario, en estudiantes que ingresan a la Universidad Autónoma de Nuevo León", en el que se informó que el desequilibrio psíquico medido por el MMPI resultó ser un predictor inadecuado del rendimiento académico (Moral de la Rubia, 2003).

Otros reportes que definitivamente no apoyan la validez de los rasgos de personalidad como predictores de rendimiento académico son los estudios de Benbassat J, Baumal R. (2007), de Lievens F, Coetsier P, De Fruyt F, De Maeseneer J. (2002), y los trabajos de investigación efectuados por Kulasegaram K, Reiter HI, Weisner W, Hacket RD (2010).

Desde luego, los rasgos de personalidad no pueden considerarse como el único factor incidente en el rendimiento académico, existen otros factores que han sido estudiados por investigadores tales como Celis (1986), Jimenez, David, Guevara (2008), Di Gresia, Porto, Ripani (2002), Edel (2003), Extremera y FernándezBerrocal (2004), Ferguson, James y Madeley (2002), Gaxiola, González, Contreras y Gaxiola (2012), Glasser (1985), Navarro (1997), Porcel, Dapozo, y López (2010), Vélez y Roa (2005) entre muchos otros más.

Sin embargo dichos trabajos no son concluyentes en cuanto a cuáles factores son los que tienen el mayor peso explicativo en relación al rendimiento académico e inclusive se tienen resultados contradictorios entre una investigación y otra, o bien en un nivel escolar los factores obtienen valores significativo mientras en otro nivel escolar confirman las hipótesis nulas dejando así la cuestión de fondo sin resolver.

En razón de estos resultados contradictorios, se realizó la presente investigación con el objetivo de conocer con mayor precisión sí los rasgos de personalidad 
medidos por el Inventario Multifacético de Personalidad de Minnesota (MMPI-II) pueden ser utilizados como predictores del rendimiento académico, además de verificar su nivel de eficacia en dicha capacidad predictiva, y comprobar además si otros factores tienen mayor peso explicativo sobre el desempeño académico.

\section{MÉTODO}

\section{DISEÑO DE INVESTIGACIÓN}

La presente investigación tuvo un diseño Ex Post Facto Tipo Correlacional, debido a que se trató de una búsqueda sistemática empírica, en la cual no se tuvo control directo sobre las variables independientes, porque ya habían acontecido sus manifestaciones o por ser intrínsecamente no manipulables. Se hacen inferencias sobre las relaciones de ellas, sin intervención directa, a partir de la variación concomitante de las variables independientes y dependientes (Kerlinger, 1984).

Los estudios correlacionales se distinguen de los descriptivos principalmente en que, mientras estos últimos se centran en medir con precisión las variables individuales, los estudios correlaciónales evalúan el grado de relación entre dos o más variables (Cancela y otros, 2010).

\section{GRUPO SOCIAL, POBLACIÓN Y MUESTRA}

Los instrumentos de estudio fueron aplicados a una muestra aleatoria conformada por 236 alumnos universitarios de todas las licenciaturas y turnos de una Universidad Privada de la ciudad de Tijuana Baja California cuya población estimada en licenciatura al momento del estudio fue de 920 estudiantes.

La muestra fue dividida en tres grupos en relación a la variable dependiente "Rendimiento Académico", agrupándose así en alumnos de Bajo Rendimiento (41.3\%), Medio Rendimiento (42.5\%) y Alto Rendimiento (15.6\%). La diferencia en los porcentajes de cada grupo se debe a que la muestra se seleccionó de forma no probabilística sin conocer de antemano el desempeño académico de los alumnos.

La muestra quedó integrada para su estudio por un $41.02 \%$ de hombres y un $58.98 \%$ de mujeres, porcentajes similares a la cantidad total de hombres y mujeres que estudian en la Universidad en estudio con un rango de edades que van de los 17 a los 25 años en una proporción superior al 90\%.

\section{OPERACIONALIZACIÓN DE VARIABLES}

\section{Variable dependiente.}

La variable dependiente para cada uno de los modelos estimados se refiere al rendimiento académico obtenido en cada una de las asignaturas cursadas por el alumno en la preparatoria, así como una medida agregada del rendimiento global del total de asignaturas cursadas por el alumno durante toda la preparatoria. 


\section{Variable Independiente.}

Los rasgos de personalidad medidos por el Inventario Multifacético de la Personalidad de Minnesota (MMPI-II) en su formato de Escalas, junto con el factor de distorsión. Los términos de interacción entre los rasgos de personalidad, edad y el sexo. Para la medición operacional del perfil de personalidad se considerarán la suma total de las puntuaciones obtenidas en las diez (10) escalas clínicas del MMPI-II y la puntuación en particular de cada una de las escalas de Validez, Clínicas, de Contenido y Suplementarias que mide el MMPI-II.

\section{Variables de Control.}

Los factores contextuales de posible incidencia en el rendimiento académico, tales como los Psicológicos (actividad mental), Sociológicos, Fisiológicos y los factores Pedagógicos, medidos mediante un instrumento de construcción propia expresamente para esta investigación denominado Factores de Incidencia en el Aprendizaje (FIA). Así como las variables personales tales como edad y sexo.

\section{INSTRUMENTOS}

Como instrumentos se utilizaron dos cuestionarios que utilizan escalas de medición cuantitativa, uno para medir los rasgos de personalidad y el segundo para evaluar Factores de Incidencia en el Aprendizaje:

I.-El Inventario Multifacético de la Personalidad de Minnesota (MMPI-II) que fue estandarizado originalmente en los Estados Unidos y después fue estandarizado para México y América Latina por la Universidad Nacional Autónoma de México (Lucio y Reyes-Lagunes, 1994).

II.- Se aplicó un segundo cuestionario construido expresamente para la presente investigación denominado Factores de Incidencia en el Aprendizaje (FIA), que medirá algunas variables de tipo contextual que también puedan influir en el rendimiento académico, tales como: Factores Psicológicos (Actividad Mental), Factores Sociológicos, Factores Fisiológicos y Factores Pedagógicos.

\section{ANÁLISIS DE LOS DATOS}

El análisis de los datos se dividió en las siguientes etapas:

\section{Aplicación de los instrumentos}

El instrumento principal (MMPI-II) y el instrumento de control (FIA-I) se aplicaron por computadora en los laboratorios de computo en la Universidad Privada en estudio con el apoyo del personal de esa área y bajo la supervisión y vigilancia del Investigador principal y los Investigadores auxiliares. El software estuvo conectado en red a una base de datos central, misma que se respaldó de forma continua y al final del proceso de la aplicación de los instrumentos la base de datos se exportó en formato Excel. 


\section{Análisis Estadístico}

La base de datos obtenida se importó al software SPSS y fue procesada para la obtención de los siguientes resultados estadísticos: Frecuencias, Medias, Modas, Desviación Estándar, Varianza y demás estadísticas descriptivas de cada uno de los factores analizados. Se obtuvieron tablas cruzadas descriptivas con las diferentes variables explicativas y de control. Se efectuó un análisis comparativo a dos grupos (Bajo Rendimiento vs Alto rendimiento) de los resultados arrojados por los dos instrumentos; MMPI-II y FIA-I, y se efectuó la validación de la compatibilidad de ambos instrumentos, y confiabilidad del FIA-I. Se realizó un análisis de regresión lineal simple y multifactorial para responder a los objetivos e hipótesis planteados. Finalmente se Interpretaron los resultados estadísticos y elaboraron las conclusiones.

\section{RESULTADOS}

\section{APLICACIÓN DE LOS INSTRUMENTOS}

El cuestionario denominado Inventario Multifacético de la Personalidad de Minnesota (MMPI-II) utilizado para medir rasgos de personalidad y el cuestionario Factores de Incidencia en el Aprendizaje (FIA), así como la ficha de captura de datos biográficos se integraron en una sola aplicación informática que se denominó Inventario Psicopedagógico Universitario (IPU) que fue contestado por un total de 236 alumnos de la Universidad Privada en estudio, en sus laboratorios de computo en el período correspondiente del 20 de Julio al 15 de agosto del 2014.

Después de concluida la aplicación del Inventario (IPU), se recuperó la base de datos y se procedió a la calificación por plantillas computarizadas de los resultados individuales del MMPI-II para la obtención de las puntuaciones de todas las escalas consideradas para su evaluación, puntuaciones en bruto que a su vez fueron convertidas a valores T para su mejor manejo y análisis estadístico.

Posteriormente se efectuó una revisión de los Perfiles de Personalidad arrojados por el MMPI-II a fin de discriminar aquellos considerados definitivamente inválidos por excederse en más de 100 puntos T en las escalas de validez $F$ (Infrecuencia) como en la Fpo (Infrecuencia Posterior), que miden respuestas al azar, contradictorias o constantes (por ejemplo todo Cierto) efectuadas por el alumno.

Al respecto, del total de las aplicaciones se descartaron como inválidos los perfiles MMPI-II de 57 alumnos, mismos que sí se seguían considerando dentro de la muestra, generaban valores extremos o atípicos en los resultados y sesgaban la Distribución de Normalidad de la propia muestra así como el respectivo análisis estadístico, por lo cual el total de cuestionarios considerados como válidos para su procesamiento estadístico quedó en 179.

\section{PRUEBA ALPHA DE CRONBACH}

Si bien el MMPI-II cuenta con una extensa historia de investigaciones enfocadas a su validación y confiabilidad, el cuestionario Factores de Incidencia en el Aprendizaje (FIA) fue construido expresamente para esta investigación con el propósito de tener un mayor control sobre algunas variables explicativas. Dicho 
cuestionario incluyó las escalas: I. Factores Fisiológicos, II. Factores Sociológicos, III. Factores Pedagógicos y IV. Factores Psicológicos (Actividad Mental). Y en el caso de la escala Factores Psicológicos (Actividad Mental) se incluyeron 4 subescalas: Concentración, Observación, Memoria y Motivación.

En razón de ello, dicho cuestionario (FIA) fue sometido a la Prueba Alpha de Cronbach (Cronbach, 1951) mediante el software SPSS versión 19 de la IBM para conocer su fiabilidad y consistencia, arrojando como resultado un valor de significancia $(p)$ de .897 , y en el caso del Alpha de Cronbach de elementos tipificados se obtuvo un valor de .916 (Tabla 1), siendo dichos valores superiores a .80 que es el mínimo aceptable de validez, por lo cual se consideró que el cuestionario FIA cuenta con un adecuado nivel de fiabilidad.

Tabla 1

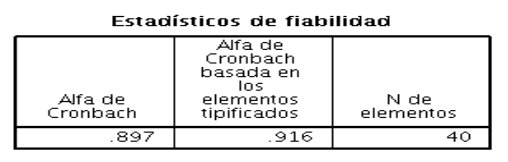

Adicionalmente se hizo una revisión de cada una de las preguntas y pudo observarse que ninguno de ellas al ser eliminadas del cuestionario cambiaba significativamente la Media, la Varianza y la Correlación Item-Total de la muestra, así como tampoco elevaba significativamente el valor total de la prueba Alpha de Cronbach

Asimismo, se obtuvieron los valores de la Media y la Desviación estándar de las escalas del Cuestionario de Factores de Incidencia en el Aprendizaje (FIA) (tabla 2) con el propósito de convertir los resultados brutos a valores $T$, para su mejor manejo estadístico y los análisis comparativos con las escalas del MMPI-II.

Tabla 2. Estadísticas Descriptivas de Factores de Incidencia en el aprendizaje.

\begin{tabular}{|l|r|r|r|r|r|}
\hline & $N$ & Mínimo & Máximo & Media & $\begin{array}{c}\text { Desvia- } \\
\text { ción Es- } \\
\text { tandar }\end{array}$ \\
\hline ACTIVIDAD MENTAL & 65 & 32 & 88 & $\mathbf{6 2 . 0 2}$ & 15.243 \\
CONCENTRACION & 65 & 7 & 26 & $\mathbf{1 6 . 5 4}$ & 5.003 \\
OBSERVACION & 65 & 7 & 26 & $\mathbf{1 5 . 6 9}$ & 5.043 \\
MEMORIA & 65 & 7 & 26 & $\mathbf{1 7 . 0 9}$ & 4.152 \\
MOTIVACION & 65 & 7 & 22 & $\mathbf{1 2 . 6 9}$ & 3.984 \\
FISIOLOGICOS & 65 & 4 & 13 & $\mathbf{8 . 5 1}$ & 2.353 \\
SOCIOLOGICOS & 65 & 4 & 18 & $\mathbf{1 0 . 0 5}$ & 2.747 \\
PEDAGOGICOS & 65 & & 14 & $\mathbf{8 . 4 6}$ & 1.977 \\
Validos & & & & \\
\hline
\end{tabular}

\section{ESTADÍSTICAS DESCRIPTIVAS DE LA MUESTRA.}

La muestra fue dividida en tres grupos independientes en relación a la variable dependiente "Rendimiento Académico" que operacionalmente fue definida en términos del Promedio General ponderado de aprovechamiento obtenido durante toda la etapa de estudios de Bachillerato del alumno previo a su ingreso a la Universidad. Dichos grupos se formaron a partir de una selección al azar de 236 
alumnos (179 perfiles válidos) de la Universidad Privada en estudio, cuyas estadísticas descriptivas resultaron las siguientes:

Tabla 3. NIVEL DE RENDIMIENTO ACADÉMICO

\begin{tabular}{|l|c|c|}
\hline \multicolumn{1}{|c|}{ GRUPO } & PORCENTAJE & ACUMULADO \\
\hline BAJO RENDIMIENTO & $41.3 \%$ & $41.6 \%$ \\
\hline MEDIO RENDIMIENTO & $42.5 \%$ & $84.3 \%$ \\
\hline ALTO RENDIMIENTO & $16.2 \%$ & $100 \%$ \\
\hline
\end{tabular}

Se puede observar en la tabla 3 , que un porcentaje muy bajo de alumnos conformaron el grupo de alto rendimiento académico (15.6\%) durante sus estudios de Bachillerato, mientras que la recepción de alumnos dentro del grupo de Bajo rendimiento fue del $41.3 \%$.

Dichas estadísticas son aún más significativas en el caso de los alumnos varones ya que el porcentaje de aquellos que tuvieron un alto rendimiento baja a tan solo $11 \%$ y aumenta el porcentaje de alumnos que tuvieron un bajo rendimiento a $54.8 \%$ como podemos apreciarlo en la tabla 4.

Tabla 4. Contingencia NIVEL DE RENDIMIENTO ACADEMICO * SEXO

\begin{tabular}{|l|l|r|r|}
\hline \multicolumn{3}{|c|}{ SEXO } & \multicolumn{1}{c|}{ MASCULINO } \\
\hline & \multicolumn{2}{|c|}{ FEMENINO } \\
\hline BAJO RENDIMIENTO & Recuento & 40 & 34 \\
& \% dentro de SEXO & $54.8 \%$ & $32.4 \%$ \\
\hline MEDIO RENDIMIENTO & Recuento & 25 & 51 \\
& \% dentro de SEXO & $\mathbf{3 4 . 2} \%$ & $\mathbf{4 8 . 6 \%}$ \\
\hline ALTO RENDIMIENTO & Recuento & $11.0 \%$ & 20 \\
& \% dentro de SEXO & & $19.0 \%$ \\
\hline $\begin{array}{l}\text { Recuento } \\
\text { \% dentro de SEXO }\end{array}$ & 73 & & 105 \\
\hline
\end{tabular}

En el caso de las mujeres, las estadísticas cambian y con ellas sube el número de alumnas con alto rendimiento (19\%) y disminuye el porcentaje en el caso de las mujeres con bajo rendimiento (32.4\%), por lo que de entrada podemos observar aquí que la variable de control Sexo, tiene una influencia inicial que deberemos someter a prueba de hipótesis. En la gráfica 1 podemos observar con más detalle la diferencia en cuanto al rendimiento académico entre hombres y mujeres.

\section{Gráfica 1.}

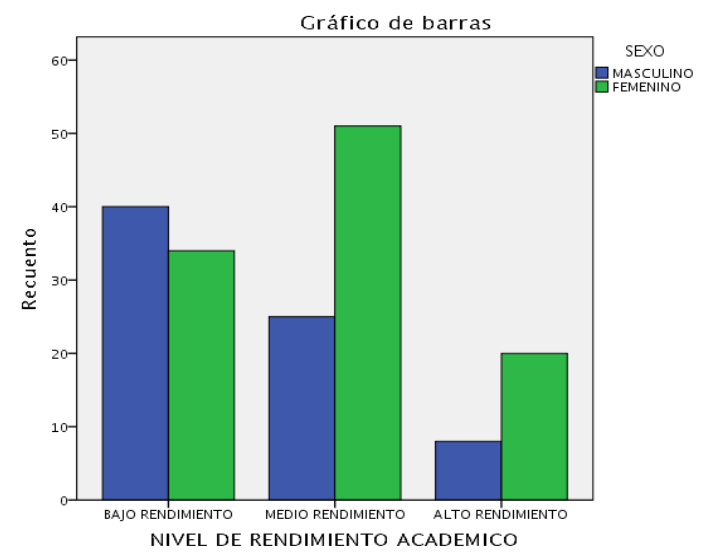




\section{ANÁLISIS COMPARATIVO A DOS GRUPOS}

Con el propósito de hacer una discriminación inicial con respecto a la existencia de diferencias significativas en las Medias entre los dos grupos de alumnos a estudio: 1. Bajo Rendimiento y 2. Alto Rendimiento, se efectuó la prueba T de Medias con Grupos Independientes (SPSS 19) con un intervalo del .95 de confianza.

De un total de 43 escalas del MMPI-II evaluadas, solamente seis (6) de ellas obtuvieron un valor de $p$ menor a .05 (Solamente el $13.5 \%$ de las escalas) y por lo tanto significativo para considerar diferencias en los valores de las medias en ambos grupos. Es decir, en este caso el nivel de eficacia no alcanza el $14 \%$ como instrumento predictor.

Las escalas que obtuvieron valores significativos fueron: I. Mentira-franqueza. II. Responsabilidad social. III. Prácticas antisociales. IV. Baja autoestima. V. Dificultades en el trabajo y VI. Rechazo al tratamiento. Mismas que pasaron a su vez la prueba de Homogeneidad de Varianza de Levene en la que todas tienen un valor de $p$ (significancia) mayor a 05. y por lo tanto se acepta la hipótesis nula de que la varianza es igual en ambos grupos: Bajo y Alto rendimiento.

Sin embargo al aplicar a estas escalas la prueba $Z$ de Normalidad de KolmogorovSmirnov con respecto a los dos grupos Alto y Bajo rendimiento, solamente la escala Prácticas antisociales y Rechazo al tratamiento obtuvieron un valor de $p$ superior a .05 y por lo tanto cumplen con el criterio de Normalidad, como lo podemos ver en la tabla 6.

Tabla 6.

\begin{tabular}{|c|c|c|c|c|c|c|}
\hline \multicolumn{7}{|c|}{ Estadísticos de contraste ${ }^{a}$} \\
\hline & & $\begin{array}{l}\text { MENTIRA- } \\
\text { FRANOUEZA }\end{array}$ & $\begin{array}{l}\text { PRACTICAS } \\
\text { ANTISOCIALE } \\
\text { S }\end{array}$ & $\begin{array}{l}\text { BAIA } \\
\text { AUTOETIMA }\end{array}$ & $\begin{array}{l}\text { DIFICULTADE } \\
\text { SEN EL } \\
\text { TRABAJO }\end{array}$ & $\begin{array}{l}\text { RECHAZOA A } \\
\text { TRATAMIENT } \\
\text { O }\end{array}$ \\
\hline \multirow{3}{*}{$\begin{array}{l}\text { Diferencias más } \\
\text { extremas }\end{array}$} & Absoluta & .438 & .240 & .346 & .303 & .290 \\
\hline & Positiva & 438 & .000 & .036 & .036 & .009 \\
\hline & Negativa & .000 & -.240 & -.346 & -.303 & -.290 \\
\hline \multicolumn{2}{|c|}{$z$ de Kolmogorov-Smirnov } & 1.975 & 1.083 & 1.557 & 1.366 & 1.305 \\
\hline \multicolumn{2}{|c|}{ Sig. asintót. (bilateral) } & .001 & 191 & .016 & .048 & .066 \\
\hline
\end{tabular}

Con respecto a las escalas del Cuestionario Factores de Incidencia sobre el Aprendizaje (FIA), solamente la correspondiente a Factores Psicológicos (Actividad Mental) obtuvo un valor menor de .05 para aceptar la hipótesis que existen diferencias significativas entre los dos grupos: Bajo Rendimiento vs Alto Rendimiento, y además cumplió la prueba de Levene de homogeneidad de varianza como lo apreciamos en la tabla 7.

Tabla 7. Prueba de Muestras Independientes

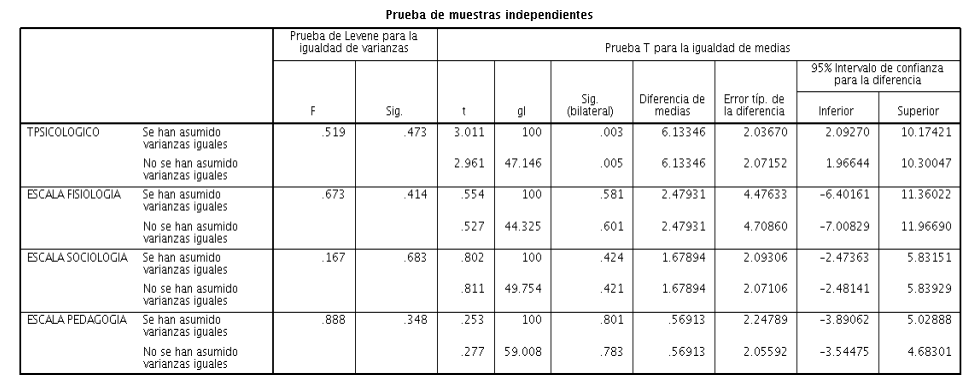


De las subescalas correspondientes a Factores Psicológicos (Actividad Mental), resultaron con un valor de significancia $(\boldsymbol{p})$ menor a .05 la referente a Concentración y la de Observación, ambas con puntuaciones superiores a .05 en la prueba de Homogeneidad de Varianza de Levene, y por lo tanto su nivel de significación es válido para rechazar la hipótesis nula y aceptar la alternativa.

Tabla 8. Prueba de Muestras Independientes

\begin{tabular}{|c|c|c|c|c|c|c|c|c|c|c|}
\hline \multicolumn{11}{|c|}{ Prueba de muestras independientes } \\
\hline & & \multicolumn{2}{|c|}{$\begin{array}{l}\text { Prueba de Levenep para la } \\
\text { igualdad de varianzas }\end{array}$} & \multicolumn{7}{|c|}{ Prueba T para la igualdad de medias } \\
\hline & & \multirow[b]{2}{*}{ F } & \multirow[b]{2}{*}{ Sig. } & \multirow[b]{2}{*}{ t } & \multirow[b]{2}{*}{$g$} & \multirow[b]{2}{*}{$\begin{array}{l}\text { Sig. } \\
\text { (bilateral) }\end{array}$} & \multirow[b]{2}{*}{$\begin{array}{l}\text { Difierencia de } \\
\text { medias }\end{array}$} & \multirow[b]{2}{*}{$\begin{array}{l}\text { Error tip. de } \\
\text { la diferenciala }\end{array}$} & \multicolumn{2}{|c|}{$\begin{array}{l}95 \% \text { Intervalo de confianza } \\
\text { para la diferencia }\end{array}$} \\
\hline & & & & & & & & & Inferior & Superior \\
\hline \multirow{2}{*}{$\begin{array}{l}\text { EECALA } \\
\text { CONCENTRACION }\end{array}$} & $\begin{array}{l}\text { Se han asumido } \\
\text { varianzas iguales }\end{array}$ & 2.828 & .096 & 4.049 & 100 & .000 & 8.36564 & 2.06610 & 4.26656 & 12.46472 \\
\hline & $\begin{array}{l}\text { No se han asumido } \\
\text { varianzas iguales }\end{array}$ & & & 4.391 & 57.904 & .000 & 8.36564 & 1.90523 & 4.55176 & 12.17951 \\
\hline \multirow[t]{2}{*}{\begin{tabular}{|l} 
EECALA OBSERVACION \\
\end{tabular}} & $\begin{array}{l}\text { Se han asumidido } \\
\text { varianzas iguales }\end{array}$ & .198 & .657 & 3.046 & 100 & .003 & 6.23353 & 2.04618 & 2.17397 & 10.29310 \\
\hline & $\begin{array}{l}\text { No se han asumido } \\
\text { varianzas iguales }\end{array}$ & & & 3.006 & 47.482 & .004 & 6.23353 & 2.07343 & 2.06344 & 10.40362 \\
\hline \multirow[t]{2}{*}{ ETCALA MEMORIA } & $\begin{array}{l}\text { Se han asumido } \\
\text { varianzas iguales }\end{array}$ & .005 & .942 & 1.126 & 100 & .263 & 2.36123 & 2.09732 & -1.79979 & 6.52225 \\
\hline & $\begin{array}{l}\text { No se han asumido } \\
\text { varianzas iguales }\end{array}$ & & & 1.108 & 47.234 & .273 & 2.36123 & 2.13109 & -1.92541 & 6.64786 \\
\hline \multirow[t]{2}{*}{\begin{tabular}{|l} 
ECALAMOTIVACION \\
\end{tabular}} & $\begin{array}{l}\text { Se han asumido } \\
\text { varianzas iguales }\end{array}$ & .006 & .937 & 1.714 & 100 & .090 & 3.14705 & 1.83561 & -49475 & 6.78885 \\
\hline & $\begin{array}{l}\text { No se han asumido } \\
\text { varianzas iguales }\end{array}$ & & & 1.762 & 51.514 & .084 & 3.14705 & 1.78572 & -.43706 & 6.73115 \\
\hline
\end{tabular}

\section{ANÁLISIS COMPARATIVO A TRES GRUPOS.}

La prueba $\mathbf{T}$ nos permite compara las Medias de dos grupos como lo hemos efectuado previamente, sin embargo como se recordara la muestra de estudio se dividió en tres grupos:

1. Bajo rendimiento (Puntuaciones $\mathrm{T}$ superiores a 50)

2. Medio rendimiento (Puntuaciones T entre 38 y 50)

3. Alto Rendimiento (Puntuaciones T igual o menores a 37)

Razón por la cual se procedió a la aplicación de la prueba ANOVA de KruskalWallis para tres o más grupos independientes cuyo propósito es aceptar o rechazar la hipótesis nula con respecto a las diferencias entre los grupos.

Los resultados arrojaron rechazo de hipótesis nula solamente en el caso de la escala Dificultades en el trabajo al tener en la prueba ANOVA un valor de significancia menor a .05 .

En el caso de las escalas del cuestionario de Factores de Incidencia en el Aprendizaje (FIA), al aplicarles la prueba ANOVA de Kruskal-Wallis, todas sus Escalas resultaron con aceptación de la hipótesis nula.

De acuerdo a los resultados antes descritos, al hacer un análisis comparativo a tres grupos se encontró que no existen diferencias significativas entre los mismos, tanto con respecto a las escalas del MMPI-2 como las escalas del cuestionario FIA.

\section{ANÁLISIS DE REGRESIÓN MÚLTIPLE LINEAL}

Al efectuar el Análisis de Regresión Múltiple Lineal incluyendo todas las escalas en estudio del MMPI-II mediante el método de pasos sucesivos, los resultados indican que solamente las escalas de Responsabilidad social (RS) y de Personali- 
dad Tipo A pueden quedar incluidas dentro del modelo, en tanto el resto de las escalas quedan excluidas por su bajo nivel de significancia dentro del análisis.

or.

De acuerdo a estos resultados del análisis de regresión lineal, el MMPI-II tiene un nivel de eficacia como herramienta predictor del rendimiento académico de apenas el $4.65 \%$, lo cual puede considerarse como insuficiente para justificar el uso del instrumento para fines psicopedagógicos en las universidades.

En cuanto a las escalas del Cuestionario Factores de Incidencia en el Aprendizaje (FIA) que se utilizaron como variables de control, el análisis de regresión lineal solamente aceptó las escalas de Concentración y Pedagogía, dejando fuera del modelo al resto de las escalas debido a que sus valores $p$ (significancia) resultaron mucho mayoror.es que .05.

Un tercer análisis de regresión incluyó de manera combinada las escalas del MMPI-II: Responsabilidad social (RS) y la escala de Personalidad Tipo A (PTA), así como las escalas del cuestionario de Factores de Incidencia en el Aprendizaje (FIA) : Concentración y Pedagogía, las cuales todas fueron aceptadas dentro del modelo de regresión con valores de $p$ (significancia) mucho menores a .05.

En el análisis comparativo a dos grupos (Bajo Rendimiento vs Alto Rendimiento) solamente seis escalas del MMPI-II resultaron con rechazo de hipótesis nula, siendo estas además de Responsabilidad social, Mentira-franqueza, Prácticas antisociales, Baja autoestima, Dificultades en el trabajo y Rechazo al tratamiento, mismas que fueron incluidas en un tercer análisis de regresión lineal, sin embargo en el método por pasos, estas también fueron finalmente excluidas del modelo.

En cuanto a las variables de control biográficas, las estadísticas descriptivas de la muestra nos indican que los hombres cuentan con un mayor porcentaje dentro del grupo de bajo rendimiento, por lo que se procedió a efectuar una prueba de hipótesis en relación al sexo y su influencia en el rendimiento académico, arrojando como resultado un nivel de significancia $(p)$ de .003 aceptándose así la hipótesis alternativa, es decir, en este caso con respecto a la muestra estudiada, si existe diferencia en cuanto al sexo en lo referente a rendimiento académico.

En el caso de la edad, la prueba de correlación arrojó un valor de $p$ igual a .946, muy superior para aceptar la hipótesis alternativa, por lo que se considera que la edad, en este caso, no influye en el rendimiento académico, particularmente porque se está tomando como base el promedio general ponderado del bachillerato.

En cuanto al Cuatrimestre al que pertenecen los alumnos la prueba de Chi-Square sobre la independencia de grupos (Cuatrimestre vs Rendimiento Académico) arrojó un valor de $\mathbf{3 4 3}$ por lo que también se acepta la hipótesis de que ambos grupos son finalmente independientes y no ejercen influencia uno sobre el otro (Tabla 9). 
Tabla 9. Chi-Square Tests

\begin{tabular}{|l|r|r|r|}
\hline & \multicolumn{1}{|c|}{ Value } & \multicolumn{1}{|c|}{$\mathrm{df}$} & $\begin{array}{r}\text { Asymp. Sig. } \\
\text { (2-sided) }\end{array}$ \\
\hline $\begin{array}{l}\text { Pearson Chi- } \\
\text { Square }\end{array}$ & $17.682^{\mathrm{a}}$ & 16 & .343 \\
Likelihood Ra- \\
tio
\end{tabular}

Por lo cual el modelo de regresión múltiple lineal final validado y significativo en este estudio quedó de la siguiente manera:

Siendo:

$$
R A=C+P+R S+P T A+S+O F
$$

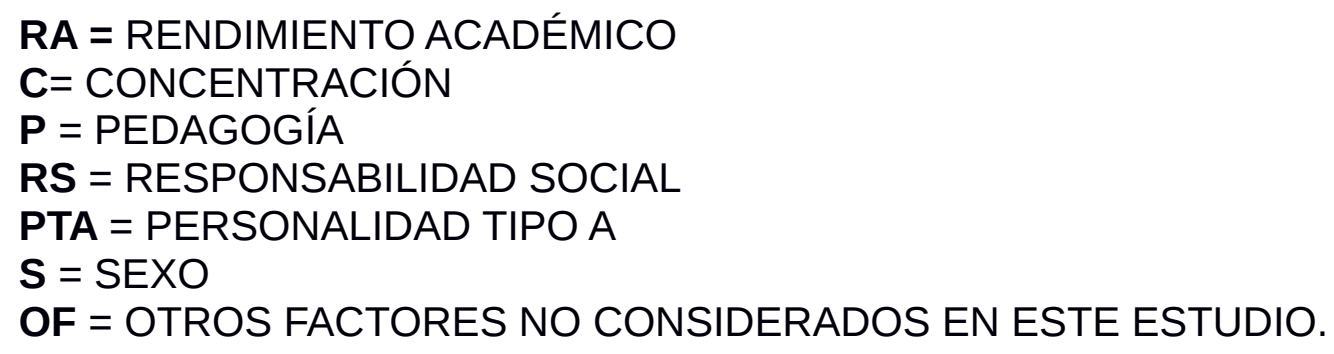

\section{PERFIL DE LOS ALUMNOS DE BAJO RENDIMIENTO}

La última parte del presente análisis es obtener el perfil de identificación de los alumnos de Bajo Rendimiento. En las tablas 10.a, 10b, 10c y 10d se puede observar las Medias de ambos grupos en cada uno de los factores que resultaron significativos en su influencia con respecto al rendimiento académico. En este caso el color rojo marrón indica qué grupo es el que presenta puntuaciones más altas con respecto al factor analizado.

Tabla 10.a Contraste Nivel de rendimiento vs Escala MMPI

\begin{tabular}{|c|c|c|}
\hline & RESPONSABILIDAD SOCIAL \\
\hline & & Media \\
\hline NIVEL DE RENDIMIENTO & BAJO RENDIMIENTO & 45 \\
\hline ACADÉMICO & MEDIO RENDIMIENTO & 49 \\
\hline & ALTO RENDIMIENTO & 54 \\
\hline
\end{tabular}

Tabla 10.b Contraste Nivel de rendimiento vs Escala MMPI

\begin{tabular}{|c|c|c|}
\hline & PERSONALIDAD TIPO A \\
\hline & & Media \\
\hline NIVEL DE RENDIMIENTO & BAJO RENDIMIENTO & 54 \\
\hline ACADÉMICO & MEDIO RENDIMIENTO & 52 \\
\hline & ALTO RENDIMIENTO & 53 \\
\hline
\end{tabular}

Tabla 10.c Contraste Nivel de rendimiento vs Escala MMPI

\begin{tabular}{|c|c|c|}
\hline & ESCALA CONCENTRACION \\
\hline & & Media \\
\hline \multirow[t]{3}{*}{$\begin{array}{l}\text { NIVEL DE RENDIMIENTO } \\
\text { ACADÉMICO }\end{array}$} & $\begin{array}{l}\text { BAJO } \\
\text { ACADÉMICORENDIMIENT } \\
\mathrm{O}\end{array}$ & 49.63 \\
\hline & MEDIO RENDIMIENTO & 47.48 \\
\hline & ALTO RENDIMIENTO & 41.27 \\
\hline
\end{tabular}




\begin{tabular}{|c|c|c|}
\hline & & $\begin{array}{c}\text { ESCALA } \\
\text { PEDAGOGIA }\end{array}$ \\
\hline & & Media \\
\hline \multirow{3}{*}{$\begin{array}{l}\text { NIVEL DE RENDIMIENTO } \\
\text { ACADÉMICO }\end{array}$} & BAJO RENDIMIENTO & 45.87 \\
\hline & MEDIO RENDIMIENTO & 46.96 \\
\hline & ALTO RENDIMIENTO & 45.30 \\
\hline
\end{tabular}

Comparando las medias de cada escala, se puede entonces efectuar una interpretación ideográfica de los resultados y construir así el Perfil Identificador de Alumnos que pertenecen al grupo de Bajo Rendimiento.

\section{Perfil Ideográfico Identificador de Alumnos de Bajo Rendimiento.}

Uno de los objetivos de la presente investigación fue el obtener un perfil promedio de los alumnos de bajo rendimiento, sin embargo como lo indican los resultados, no es posible tener un perfil clínico, de contenido o complementario del MMPI-II, debido a que solo dos escalas de dicho inventario (solamente el $4.65 \%$ de las escalas) correlacionan significativamente con bajo rendimiento académico.

Pero pese a lo anterior, sí es posible construir un perfil diferente con algunas de las escalas del MMPI-II, que den pauta a la construcción de un nuevo instrumento de medición con mucho menos preguntas y con menores requerimientos para su aplicación e interpretación.

En razón de ello, se expone en la tabla 11 las medias obtenidas en las escalas referidas y sus respectivas desviaciones estándar como perfil promedio de los alumnos de bajo rendimiento evaluados en la presente investigación.

Tabla 11. Estadísticos descriptivos ${ }^{\mathrm{a}}$

\begin{tabular}{|l|r|r|r|}
\hline & $\mathrm{N}$ & \multicolumn{1}{|c|}{ Media } & \multicolumn{1}{c|}{ Desv. típ. } \\
\hline RESPONSABILIDAD SOCIAL & 74 & $\mathbf{4 5 . 3 8}$ & $\mathbf{1 1 . 2 5 6}$ \\
PERSONALIDAD TIPO A & 74 & $\mathbf{5 4 . 3 6}$ & $\mathbf{1 0 . 8 8 7}$ \\
MENTIRA-FRANQUEZA & 74 & $\mathbf{6 1 . 3 2}$ & $\mathbf{1 2 . 3 5 3}$ \\
BAJAAUTOESTIMA & 74 & $\mathbf{5 3 . 6 4}$ & $\mathbf{9 . 0 8 2}$ \\
PRÁCTICAS ANTISOCIALES & 74 & $\mathbf{5 7 . 2 4}$ & $\mathbf{8 . 6 6 7}$ \\
DIFICULTADES EN EL & 74 & $\mathbf{5 4 . 9 2}$ & $\mathbf{1 1 . 0 1 0}$ \\
TRABAJO & & & \\
RECHAZO A TRATAMIENTO & 74 & $\mathbf{5 6 . 9 1}$ & $\mathbf{1 0 . 6 5 5}$ \\
N válido (según lista) & 74 & & \\
\hline
\end{tabular}

Y adicionalmente también se incluye aquí la tabla 12 con los valores promedio de las escalas que tuvieron una correlación significativa en cuanto al Cuestionario Factores de Incidencia en el Aprendizaje (FIA), como perfil de los alumnos de bajo rendimiento.

Tabla 12. Estadísticos descriptivos ${ }^{\mathrm{a}}$

\begin{tabular}{|l|r|r|r|}
\hline \multicolumn{2}{|c|}{$\mathrm{N}$} & \multicolumn{1}{|c|}{ Media } & \multicolumn{1}{c|}{ Desv. típ. } \\
\hline ESCALA CONCENTRACIÓN & 74 & $\mathbf{4 9 . 6 3 1 4}$ & 9.71633 \\
ESCALA OBSERVACIÓN & 74 & $\mathbf{4 8 . 4 0 5 7}$ & 9.14871 \\
ESCALA PEDAGOGÍA & 74 & $\mathbf{4 5 . 8 7 2 7}$ & $\mathbf{1 0 . 6 1 1 0 3}$ \\
N válido (según lista) & 74 & & \\
\hline
\end{tabular}


Finalmente se concluye el análisis de resultados presentando la interpretación ideográfica del perfil promedio antes expuesto en las tablas 11 y 12 correspondientes a la muestra de alumnos de bajo rendimiento:

a. Son alumnos con un nivel de Responsabilidad Social más alto que los demás alumnos, por lo que pueden prestar más atención en resolver los problemas que enfrentan sus familiares, amigos o compañeros de trabajo o estudios, que en estudiar o hacer sus tareas y actividades de aprendizaje, o bien pueden tener un nivel de integridad académica más alto que sus compañeros y tienden a no copiar o hacer trampa en los exámenes.

b. Son alumnos que enfrentan serios problemas de Concentración en sus clases y actividades de aprendizaje, por lo que tienden a distraerse con mayor facilidad, dejan de prestar atención y terminan por desconectarse del tema en estudio dentro de la clase o de la lectura.

c. Son alumnos que consideran que sus profesores no cuentan con las competencias Pedagógicas o la capacitación necesaria para enseñar o piensan que los maestros no saben manejar apropiadamente la didáctica de clase.

d. Son alumnos con problemas de Personalidad tipo A. Es decir, personas con tenencia a estar siempre haciendo algo productivo con una terrible aversión a todo aquello que suene a pérdida de tiempo. Esto puede incluir el descanso o el ocio. Hacen las cosas con rapidez, se quiere acabar rápido, como si siempre tuvieran prisa. Personas con predominio de lo racional por encima de lo emocional. Actitud competitiva, con cierta tendencia a la hostilidad. Muy centrados en la carrera profesional, llegando a relegar la vida personal por ello. Dan mucha importancia al resultado, llegando a no disfrutar de verdad de los procesos que llevan a dicho resultado. Personas que tienden hablar rápido, en voz alta y con tendencia a interrumpir. Son alumnos multitarea. Tienen necesidad de controlar el ambiente. Tenacidad y perseverancia. Problemas para conciliar el sueño o sueño ligero. Personas con poca tolerancia hacia la incompetencia o lentitud de otras personas y con tendencia a hacer listas de cosas que se tienen que hacer.

e. Se trata de alumnos con una menor proclividad a mentir y a faltar a la integridad académica en los exámenes.

f. Son alumnos con un nivel más bajo de Autoestima y que con frecuencia presentan un mayor nivel de Problemas en el Trabajo que inciden en su rendimiento académico.

\section{DISCUSIÓN Y CONCLUSIONES}

El hecho de que las Universidades Privadas acepten en su matrícula un alto porcentaje de alumnos con historial de Bajo Rendimiento académico y un bajo porcentaje de estudiantes considerados como de Alto Rendimiento, le impone a las mismas una serie de problemas, retos y dificultades que deben de superarse desde un enfoque psicopedagógico que se sustente en el fortalecimiento de la vida académica, la auto evaluación institucional y el diseño de políticas, planes y 
programas encausados al apoyo, la tutoría y la nivelación de alumnos con problemas de bajo rendimiento, a fin de que dichas instituciones se mantenga en los más altos estándares de calidad educativa.

La presente investigación aporta su parte a dicho fin al analizar la posibilidad de que un inventario de amplio uso como es el MMPI-II, pueda ser utilizado como una herramienta predictor del rendimiento académico, y de esa manera los responsables de los Departamentos Psicopedagógicos en las universidades, puedan contar con la base para la definición de políticas y programas encausados a la tutoría y la nivelación académica de sus alumnos.

Por ello, luego de la revisión teórica y la consecuente investigación aplicada con diseño Ex post Facto Tipo Correlacional, es importante concluir sintetizando las interpretaciones de los principales resultados.

Pero, con el fin de mantener la coherencia seguida a lo largo de la investigación, lo más adecuado es efectuar una análisis de las hipótesis planteadas en el Protocolo de Investigación y su aceptación o rechazo, a colación de las cuales se irán determinando las conclusiones o aportaciones del estudio.

En lo que respecta a la primera Hipótesis "H1. No existen diferencias significativas en el perfil de personalidad (medido por el MMPI-II) entre los alumnos de alto rendimiento y bajo rendimiento", los resultados aceptaron la hipótesis nula, lo que indica que en cuanto a los perfiles de personalidad del MPPI-II, ya sea Clínico, De Contenido y Suplementario, estos no permiten discriminar a los alumnos de bajo rendimiento de los de alto rendimiento. En ese sentido se puede concluir que dicho inventario resulta ineficiente en conjunto para diagnosticar y predecir qué alumnos de nuevo ingreso tendrán problemas de desempeño académico durante su estancia en la universidad.

En relación a la Hipótesis "H2. Los alumnos de bajo rendimiento no presentan un número mayor de escalas del MMPI con alta puntuación que el promedio de la población", también resulto cierta, ya que en los análisis comparativos, si bien seis escalas del MMPI-II obtuvieron valores de significancia $p$ mayores a .05, los datos no indican que en promedio los alumnos de bajo rendimiento tengan más escalas con alta puntuación, ya que en realidad dichas puntuaciones se acercan más a las medias de cada escala en el Grupo Bajo Rendimiento que no son sustantivamente más altas que las medias de cada escala en el Grupo Alto Rendimiento, quitándole así al MMPI-II la posibilidad de ser utilizado como una herramienta predictor.

Sin embargo, en la hipótesis "H3. No existen escalas del MMPI-Il que estén relacionadas significativamente con el bajo rendimiento de los alumnos.", los resultados arrojaron confirmación de la hipótesis alternativa, ya que en el análisis comparativo a dos grupos (Bajo rendimiento vs Alto rendimiento) seis escalas (Mentira-franqueza, Responsabilidad social, Prácticas antisociales, Baja autoestima, Dificultades en el trabajo y Rechazo al tratamiento) del MMPI-II resultaron con niveles de significancia $(p)$ menores a .05 rechazándose así la hipótesis nula y aceptando la alternativa, y por otra parte en el análisis de regresión múltiple lineal, las escalas Responsabilidad Social y Personalidad Tipo $A$, obtuvieron valores del coeficiente de correlación altos para una significancia $(p)$ menor a .05, rechazándose también en este caso la hipótesis nula. 
Lo anterior tiene implicaciones para futuras investigaciones en el ámbito de la psicometría que permitan la construcción de un nuevo instrumento con menos preguntas y afinado con escalas que realmente estén vinculadas 0 correlacionadas significativamente con el rendimiento académico.

La hipótesis "H4. No existe un perfil de personalidad promedio que esté relacionado de forma significativa con bajo rendimiento." y la hipótesis "H5. No existe un perfil de personalidad promedio que esté relacionado de forma significativa con alto rendimiento.", tuvieron el mismo resultado que la hipótesis $\mathrm{H} 1$, se aceptó en ambos casos la hipótesis nula y por lo tanto queda de lado la posibilidad de utilizar el MMPI-II como predictor del Bajo Rendimiento o del alto rendimiento.

En lo referente a variables de control, la hipótesis "H6. Los factores de control como la edad y el sexo no inciden significativamente en relación con el perfil de personalidad, sobre el rendimiento escolar.", se aceptó la hipótesis nula en el caso de la edad, pero en el caso del sexo se aceptó la hipótesis alternativa pues este sí incide positivamente, siendo las mujeres las que presentan un mayor porcentaje como alumnas de mediano y Alto rendimiento y con menor porcentaje como alumnas de bajo rendimiento.

Finalmente en cuanto a la hipótesis "H.7 Otros factores como los psicológicos (actividad mental), Sociológicos, Fisiológicos y Pedagógicos no influyen en el Bajo rendimiento académico", se rechazó parcialmente la hipótesis Nula, debido que la escala Factores Psicológicos (actividad mental) en particular sus subescalas (Concentración, Observación) y la escala Factores Pedagógicos, resultaron con aceptación de la hipótesis alternativa.

Adicionalmente, en el análisis de regresión lineal, estos factores resultaron tener un mayor peso explicativo del bajo rendimiento académico que los rasgos de personalidad medidos por el MMP-II, lo que tiene implicaciones serias en la orientación de las investigaciones sobre las causas del bajo rendimiento, pues nos indica que las posibles explicaciones del bajo rendimiento en lugar de encontrarlas en la formación de la personalidad de los individuos, deben de ubicarse por parte de los investigadores en las fallas de la actividad mental de los alumnos enfocada al estudio (Concentración, capacidad de análisis, Observación, lectura crítica o analítica, aprendizaje pro-activo, etc. etc.), y por otra parte en las fallas que presentan los profesores con respecto al manejo pedagógico o didáctico de sus clases, aunque dados los magros resultados en la evaluación de la actividad mental de los alumnos, estos por lo mismo pueden tener una pobre opinión con respecto al desempeño de sus profesores.

\section{V.II CONCLUSIÓN GENERAL}

La presente investigación no arrojó resultados o evidencias contundentes para considerar el Inventario Multifacético de la Personalidad de Minnesota (MMPI-II) como una herramienta predictor del Rendimiento Académico, además su eficacia de predicción se ubicó abajo del 5\%, por lo tanto resulta innecesario, oneroso e inútil aplicarlo con ese propósito. 
Pero por otra parte la investigación aporta evidencias para correlacionar de manera significativa el Rendimiento académico con algunos rasgos de personalidad (Responsabilidad social, Personalidad tipo A, Mentira-franqueza, Autoestima, Prácticas antisociales, y Rechazo a tratamientos), lo que tiene implicaciones para futuras investigaciones en el ámbito de la psicometría y la personalidad.

Los Factores psicológicos (Actividad mental) y su subescala Concentración y Observación, así como los Factores Pedagógicos tuvieron un mayor peso explicativo que los rasgos de personalidad en el análisis de regresión lineal e inclusive en un análisis discriminante, lo que a su vez tiene implicaciones mucho mayores en el ámbito de futuras investigaciones sobre las causas del rendimiento académico.

\section{REFERENCIAS BIBLIOGRÁFICAS}

Benbassat J, Baumal R. (2007). Uncertaintines in the selection of applicants for medical school. Advances Health Science Education; 12:509-521.

Butcher Jn., Lim J., Nezami E. (1998). Objective study of abnormal personality in cross-cultural settings. the Minnesota Multiphasic Personality Inventory (MMMPI2). Journal of Cross-cultural Psychology. 29: 189-211.

Cancela, G.R. et al. (2010) Metodología de la Investigación educativa: Investigación Ex Post Facto. Universidad Autónoma de Madrid.

Celis, G.A. (1986). Los subtests de razonamiento abstracto, razonamiento verbal y relaciones espaciales del D.A.T., como elementos predictivos de rendimiento académico en la U.I.A.. México. Tesis de Licenciatura en Psicología. Universidad Iberoamericana.

Coppari, N. B. y colbs: estudiantes de $3^{\circ}$ y $4^{\circ}$ curso de Psicología (2001). Medición Transversal de Patrones de Comportamiento Vocacional y de Personalidad en Estudiantes de Psicología. Universidad Católica de Asunción, Facultad de Filosofía y Ciencias Humanas, Carrera de Psicología, Cátedras de Psicología Experimental I y II, Proyecto Impreso, Asunción-Paraguay.

Coppari, N. y colbs:estudiantes de cuarto curso turno noche de la Carrera de Licenciatura en Psicología (2007). Análisis de Perfiles de Personalidad de Estudiantes de Psicología. Revista Eureka, Vol. N7, pp.5-13.

Coppari, N. (2007) Análisis Multivariado Aplicado a Perfiles del MMPI-2 de Estudiantes de Psicología. Tesis de Grado para Doctorado. Universidad Católica Nuestra Señora de la Asunción.

Cronbach LJ. (1951) Coefficient alpha and the internal structure of test. Psychometrika. 16:297-334.

De León J. (1991) Rasgos de personalidad del estudiante de primer ingreso de la Escuela de Ciencias Psicológicas según el MMPI y su relación con el rendimiento académico. [tesis Licenciatura en Psicología]. Guatemala: Universidad de San 
Carlos de Guatemala. Guatemala.

Di Gresia, L., Porto, A. y Ripani, L. (2002). Rendimiento de los Estudiantes de las Universidades Públicas Argentinas. Universidad Nacional de la Plata.

Departamento de Economía. Documento de Trabajo No. 45. Disponible en: www.depeco.econo.unlp.edu.ar/doctrab/doc45.pdf

Edel, R. (2003). Factores asociados al rendimiento académico. Revista Iberoamericana de Educación. Organización de Estados Iberoamericanos para la Educación, la Ciencia y la Cultura. En red. Recuperado en: http://www.campusoei.org/revista/frame_participar.htm. Sección: Investigación, 20 de Septiembre de 2003.

Extremera, Natalio y Pablo Fernández-Berrocal (2004), "El papel de la inteligencia emocional en el alumnado: evidencias empíricas", en Revista Electrónica de Investigación Educativa, vol. 6, núm. 2.

Ferguson E, James D, Madeley L. (2002). Factors associated with success in medical school: systematic review of the literature. Br; 324:952-957.

Gaxiola R, José, Sandybell González Lugo, Zita Contreras Hernandez y Eunice Gaxiola Villa (2012). Predictores del rendimiento académico en adolescentes con disposiciones resilientes y no resilientes. Revista de Psicologia, Vol. 30, núm. 1.

Gaxiola R, José, Lucio, G-M. E. y Reyes-Lagunes, I., (1994). La nueva version del Inventario Multifasico de la Personalidad de Minnesota MMPI-2 para estudiantes universitarios Mexicanos. Revista Mexicana de Psicologia. Volumen 11, numero 2, pag. 45-54.

Glasser, W. (1985). Escuelas sin fracasos. México: Pax-México.

Hathaway S.R., Mckinley J.C. (1995) Inventario multifásico de la personalidad Minnesota 2. Manual para la aplicación y calificación. México, D.F. El Manual Moderno.

Jimenez R, David, Yolanda Guevara Benitez (2008). Comparación de dos estrategias de intervención en interacciones Madre-hijo. Su relación con el rendimiento escolar. Revista Mexicana de Análisis de la Conducta: 219-244.

Kulasegaram K, Reiter HI, Weisner W, Hacket RD et al. (2010). Non-associa-tion between Neo-5 personality tests and multiple mini-interview. Ad-vances Health Science Education Theory Practice; 15:415-423.

Lemus O. (1988). Rasgos de personalidad en estudiantes de primer ingreso a la Escuela de Ciencias Psicológicas, detectados por medio del MMPI. [tesis Licenciatura en Psicología]. Universidad de San Carlos de Guatemala. Guatemala.

Lievens F, Coetsier P, De Fruyt F, De Maeseneer J. (2002). Medical students' personality characteristics and academic performance: a five-factor model perspective. British Medical J; 326:1050-1056. 
Lucio, G-M. E. y Reyes-Lagunes, I., (1994). La nueva version del Inventario Multifasico de la Personalidad de Minnesota MMPI-2 para estudiantes universitarios Mexicanos. Revista Mexicana de Psicologia. Volumen 11, numero 2, pag. 45-54.

Martínez G.L., Nava G., Ortega M.P. (2005). Estudio exploratorio del perfil psicológico de los alumnos de nuevo ingreso a la carrera de Psicología del Centro Universitario de Ciencias de la Salud de la Universidad de Guadalajara. Revista de Educación y Desarrollo No. 4.

Moral de la Rubia J. (2003) Predicción de rendimiento académico universitario. Nuevo León México: Universidad Autónoma del Estado de México.

Navarro, Ruben Edel (1997). Factores asociados al rendimiento académico. Revista Iberoamericana de Educación.

Porcel, E., Dapozo, G. y López, M. (2010). Predicción del rendimiento académico de alumnos de primer año de la FACENA (UNNE) en función de su caracterización socioeducativa. Revista Electrónica de Investigación Educativa, 12(2).

Rios García Z. R. (2012). Rasgos de Personalidad y Rendimiento Académico. Facultad de Ciencias Médicas, Universida de San Carlos. Guatemala.

Vargas I, Ramírez C, Cortés J, Farfán A, Heinze G. (2011). Factores asociados al rendimiento académico en alumnos de la Facultad de Medicina: estudio de seguimiento a un año. Salud Mental. Jul-Ago; Numero 34, Pag. 301-8.

Vélez, A. y Roa, C. (2005). Factores asociados al rendimiento académico en estudiantes de medicina. Educación Médica, 8(2). 